\title{
Novel design of an all-cryogenic RF pound circuit
}

\author{
Basu, Ronni; Wang, R. T.; Dick, G. J.
}

Published in:

Proceedings of the 2005 IEEE International Frequency Control Symposium and Exposition, 2005.

Link to article, DOI:

10.1109/FREQ.2005.1573994

Publication date:

2005

Document Version

Publisher's PDF, also known as Version of record

Link back to DTU Orbit

\section{Citation (APA):}

Basu, R., Wang, R. T., \& Dick, G. J. (2005). Novel design of an all-cryogenic RF pound circuit. In Proceedings of the 2005 IEEE International Frequency Control Symposium and Exposition, 2005. IEEE.

https://doi.org/10.1109/FREQ.2005.1573994

\section{General rights}

Copyright and moral rights for the publications made accessible in the public portal are retained by the authors and/or other copyright owners and it is a condition of accessing publications that users recognise and abide by the legal requirements associated with these rights.

- Users may download and print one copy of any publication from the public portal for the purpose of private study or research.

- You may not further distribute the material or use it for any profit-making activity or commercial gain

- You may freely distribute the URL identifying the publication in the public portal 


\section{Novel Design of an All-Cryogenic RF Pound Circuit}

\author{
Ronni Basu \\ Department of Physics \\ The Technical University of Denmark \\ 2800 Kgs. Lyngby, Denmark \\ basu@fysik.dtu.dk
}

\author{
Rabi T. Wang and G. John Dick \\ Jet Propulsion Laboratory \\ California Institute of Technology \\ 4800 Oak Grove Drive \\ Pasadena, CA, USA
}

\begin{abstract}
We report on the design, construction and test of a new all-cryogenic RF Pound circuit used to stabilize a $100 \mathrm{MHz}$ VCXO. Here, all active and passive RF components used to accomplish the phase modulation and detect a PM to AM conversion have been installed into the cryogenic environment. In conjunction with a high- $Q$ cryogenic sapphire resonator a Pound discriminator sensitivity of $0.1 \mathrm{mV} / \mathrm{Hz}$ was seen experimentally. Based on this sensitivity and the noise properties of the pre-amplifier of the Pound signal, we calculate a limit of the oscillator's Allan deviation as low as $4 \cdot 10^{-16} / \sqrt{ } \tau$. Experimentally, the first experiments gave a stability of $1 \cdot 10^{-13}$ at 1 second.
\end{abstract}

\section{INTRODUCTION}

The Pound method [1] is widely used in oscillators with ultra-high stability [2], [3]. The central mechanism in the Pound method is illustrated in Fig. 1. Here, the spectrum of an incoming phase modulated carrier is shown on the left (only the first set of sidebands shown here). The signal that is reflected from the resonator is shown on the right. All spectral components are represented as phasors in the reference frame of the carrier. The illustration of the signal transformation is valid when the bandwidth of the resonator is much smaller than the separation in frequency between neighboring spectral components in which case only the phase and amplitude of the carrier are affected by the resonator while all the sidebands are reflected unaltered. When the carrier is off resonance the reflected carrier is phase shifted which gives rise to the partial PM to AM conversion. The AM signal is then detected and used to correct the frequency error.

The Pound circuit therefore acts as a frequency discriminator with a performance that is tightly linked to the quality factor of the resonator. As such, ultra-high stability oscillators have been achieved by using cryogenic sapphire resonators with quality factors reaching more than $10^{9}$ [2], [3]. In these implementations of the Pound method the phase modulated carrier was obtained by dithering a phaselocked DRO kept at room-temperature.

In [4], temperature variations in the microwave components between the cryogenic resonator and the roomtemperature phase modulator were analyzed theoretically

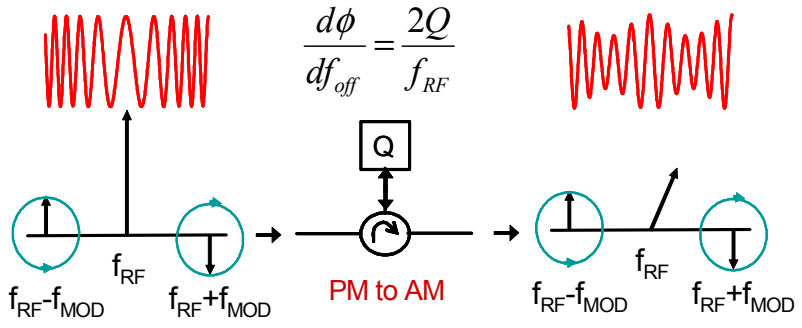

Fig. 1. Illustration of the central mechanism in the Pound stabilization method. A phase modulated carrier is applied to a resonator. The reflected signal will have an AM component if the carrier is off resonance. The AM component can be detected and used to stabilize the source of the carrier.

and shown to give rise to false Pound signals. Specifically for the oscillator described in [2], VSWR-induced standing waves in the RF transmission line going into the cryostat were estimated to give rise to a relative frequency variation of $1 \cdot 10^{-13}$ in the presence of temperature variations of $10 \mathrm{~K}$. The false Pound signals that were induced here were due to an induced linear variation of the amplitudes across the PM spectrum. Thus, [4] provided an explanation for the false Pound signals that were experimentally seen and reported in [5].

In this work we aim to eliminate the sensitivity of the oscillator to the above described temperature fluctuations in the microwave components kept at room-temperature. This is achieved by integrating all the RF components in the Pound scheme into the highly temperature stabilized environment that the sapphire resonator is placed in. Specifically, both the phase modulation of the RF carrier and the detection of the AM component are included in the cryogenic part of the oscillator. The active component in this all-cryogenic Pound circuit is the Tunnel diode which is used both as modulator and detector. This diode was chosen since it doesn't freeze out and has low noise.

We find experimentally that the new Pound circuit has a sensitivity of $0.1 \mathrm{mV} / \mathrm{Hz}$ which we calculate to set a limit on the stability as low as $4 \cdot 10^{-16} / \sqrt{ } \tau$. The preliminary

Ronni Basu would like to thank the committee of the conference for travel support 


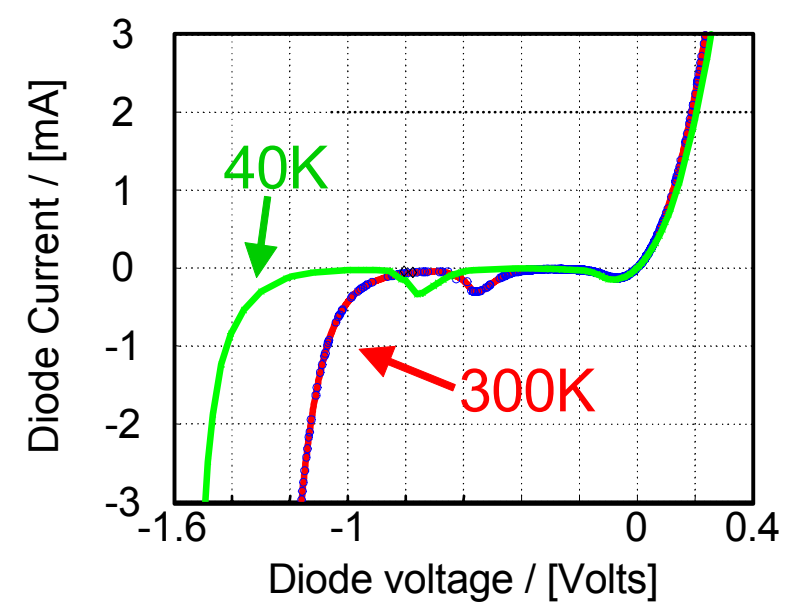

Fig. 2. IV curve of GaAs tunnel diode measured at room-temperature and $40 \mathrm{~K}$.

experimental results reported here show a stability of $1 \cdot 10^{-13}$ at 1 second.

\section{TECHNICAL}

\section{A. Tunnel Diode Model and Experiment}

The tunnel diode is a pn junction where both the $\mathrm{p}$ and the $\mathrm{n}$ part are degenerately doped. This means that the Fermi level is embedded inside the valence band of the $\mathrm{p}$ region and inside the conduction band of the $\mathrm{n}$ region. Therefore, even at $0 \mathrm{~K}$ there will be electrons in the conduction band of the $n$ region and holes in the valence band of the $p$ region, i.e. the tunnel diode does not freeze out. The distribution of electrons in the energy band diagram of the $p$ region is varying very little with temperature since the density of states drops to zero at the top of the valence band. In the $n$ region the distribution will have a high temperature dependence due to the growing $\left(\propto \sqrt{ }\left(E-E_{C}\right)\right)$ density of states. This explains the difference between the IV characteristics in Fig. 2, where positive voltages correspond to applying a positive voltage to the $\mathrm{n}$ region with respect to the $\mathrm{p}$ region. With positive voltages, valence electrons from the relatively temperature independent distribution in the $\mathrm{p}$ region is tunneling to empty states in the conduction band of the $n$ region. For negative voltages it is the conduction electrons from the temperature dependent distribution in the $\mathrm{n}$ region that are carrying the current by their tunneling into the valence band of the $\mathrm{p}$ region for small bias voltages and increasingly into the conduction band of the $\mathrm{p}$ region with increasing bias voltages. The 2 curves are measurements of a GaAs diode [6] at room-temperature and $40 \mathrm{~K}$ respectively. See [7] for a more detailed description of the tunnel diode dynamics.

The power handling ability of the tunnel diode is somewhat limited compared to other diodes so it was a concern whether an all-cryogenic Pound circuit could be

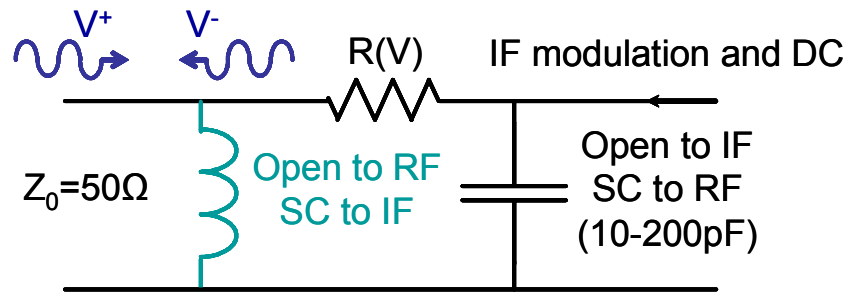

Fig. 3. Model of the tunnel diode. Left side represents the RF port and the right side the IF port.

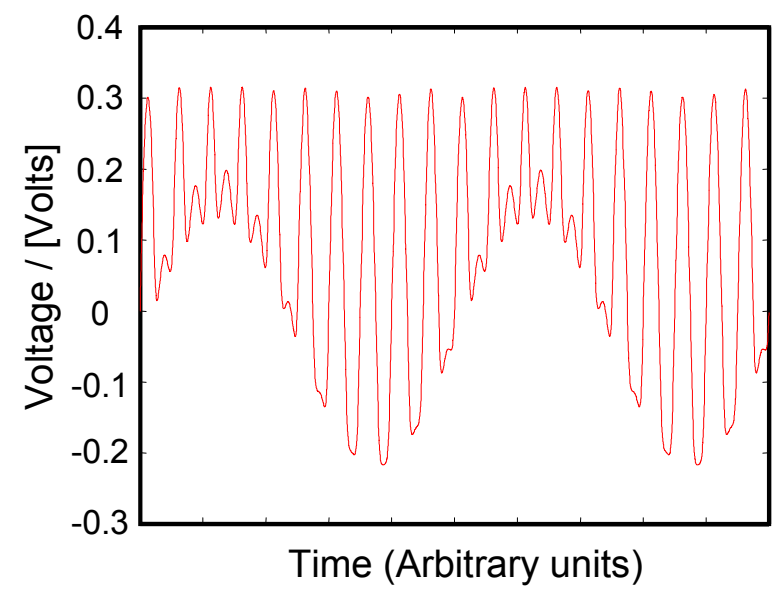

Fig. 4. Sample of the computed reflection from the RF port of the tunnel diode. The phase inversion that accounts for the carrier suppression at certain bias levels is seen where the modulation signal and the incoming RF signal add constructively.

built with them without resorting to an unwanted cold RF amplifier. Therefore, a modeling of the diode was performed initially to either confirm or deny the use of tunnel diodes for our purpose. We model the packaged diode as a simple lumped circuit shown in Fig. 3 and assume that the dynamic resistance $R(V)$ is constant over the frequency range that we are considering i.e. equal to the inverse slope of the curves in Fig. 2. The left side of the diagram represents the RF input port. The RF signal inserted here $\left(V^{+}\right)$is bypassed to ground through a capacitor on the right side of the diagram representing the IF port. Here, we generally apply both a $\mathrm{DC}$ bias $\left(D C_{B I A S}\right)$ and the harmonic modulation signal $V_{M O D}$. These signals are bypassed to ground through an inductor on the left side of the diode. Generally, $R(V)$ is different from the characteristic impedance $Z_{0}$, so a reflected wave will be generated $(V)$ at the RF port. The voltage across the tunnel diode is now simply given by:

$$
V(t)=V^{+}(t)+V^{-}(t)-V_{M O D}(t)-D C_{B I A S},
$$




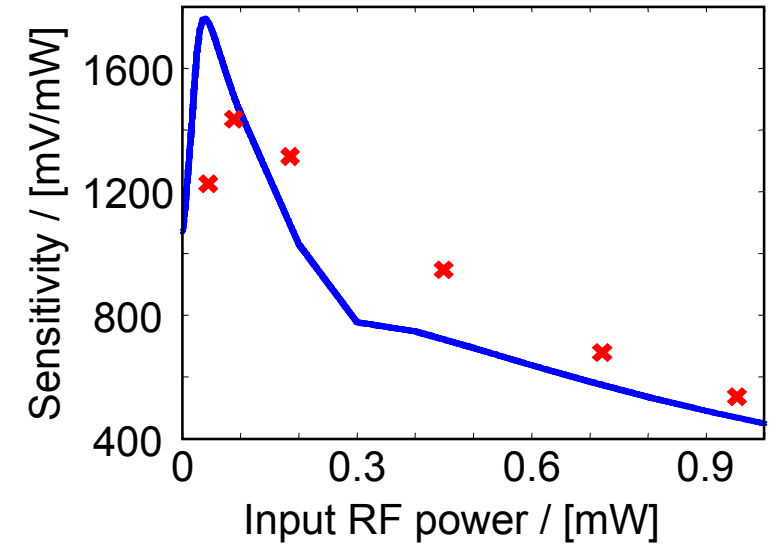

Fig. 5. The diode performance as detector. The blue curve is the calculated result based on the diode model. Red crosses are actual experimental values. The sensitivity is the ratio between the output voltage on the IF port with an open termination and the input power level at the RF port.

And Ohm's law gives us (the time dependence is implicit in the following):

$$
\frac{V^{+}+V^{-}-V_{M O D}-D C_{B I A S}}{I^{+}-I^{-}}=R(V)
$$

Here, $I^{+}$and $I$ are the incoming and outgoing current at the RF port associated with $V^{+}$and $V$, respectively. These are of course related to the characteristic impedance of the $\mathrm{RF}$ transmission line through

$$
\frac{V^{+}}{I^{+}}=\frac{V^{-}}{I^{-}}=Z_{0}
$$

Using (1), (2) and (3) we can compute the reflected signal:

$$
V^{-}=\frac{V^{+}\left[R(V)-Z_{0}\right]+Z_{0}\left[V_{M O D}+D C_{B I A S}\right]}{Z_{0}+R(V)} .
$$

$R(V)$ is a function of $V$ and as such there is only an analytical solution to (4) when $R(V)$ is well-behaved for instance around the point of origin where the tunnel diode IV curve is approximated by a second order polynomial. Outside this region, a $3^{\text {rd }}$ order time stepping scheme is used to estimate a temporary value for $V$ at the new time step. This value is used to find $R(V)$ from which $V$ is updated through (4).

In Fig. 4, a sample plot of the reflected signal is shown. The reflected signal will generally both contain a DC component $\left(V_{D C}\right)$ as well as a component having the modulation frequency $\left(V_{M O D}\right)$. These signals are unphysical due to the inductor that short low frequency signals to

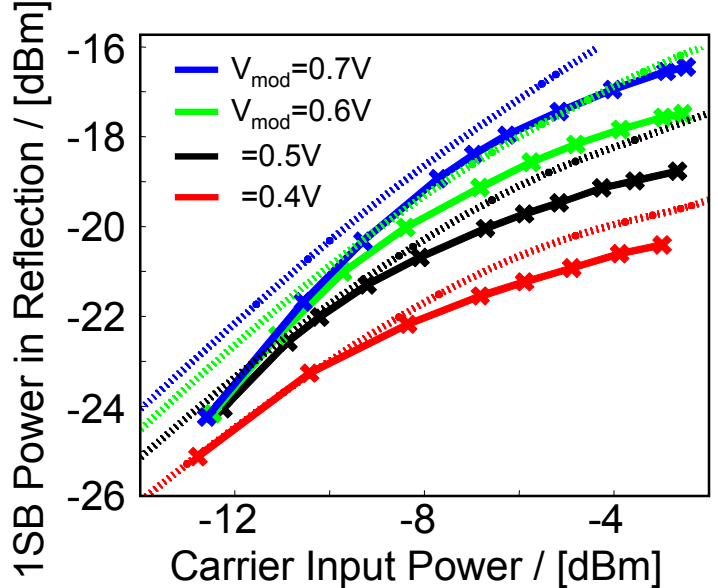

Fig. 6. The modulation ability of the tunnel diode. Dotted curves are model results, full curves are experiments. The power in one of the nearest side bands to the carrier in the reflection from the RF port is shown as function of carrier input power and at four different modulation peak-topeak voltage amplitudes applied at the IF port. The DC voltage across the diode was zero.

ground on the RF port. Therefore, once the time series of the reflected signal have been computed, and the spectral components found, the true DC level $\left(D C_{B I A S}\right.$ TRUE $)$ and the true amplitude of the modulation signal $\left(V_{0, M_{O} D_{-} T R U E}\right)$ can be computed:

$$
\begin{gathered}
D C_{B I A S_{-} T R U E}=D C_{B I A S}-V_{D C}^{-} \\
V_{0, M O D_{-} T R U E}=V_{0, M O D}-V_{M O D}^{-}
\end{gathered}
$$

where, $V_{0, M O D}$ was the amplitude of the modulation signal applied in the simulation.

Since both the RF signal and the modulation on the IF port only 'sees' the purely resistive impedance $R(V)$, the reflected RF signal is either in phase or in reverse phase with the incoming RF signal i.e. the RF reflection in the model is a purely amplitude modulated signal. To achieve the desired phase modulated signal the idea is to remove the carrier and insert it again with a 90 degree phase shift. Two different carrier suppression techniques were investigated (see next section). For now, we need to know whether a phase modulator based on the reflected AM signal from the tunnel diode along with the sapphire resonator and a tunnel diode detector will provide good enough Pound sensitivity to support further development of the oscillator. The key parameters here are 1) the amount of power available in the modulation sidebands from the diode, 2) the quality factor of the resonator, and 3) the sensitivity of the Pound detector.

In Fig. 5, the sensitivity of the tunnel diode has been shown. The model results have been obtained in simulations where the modulation is switched off on the IF port and the 


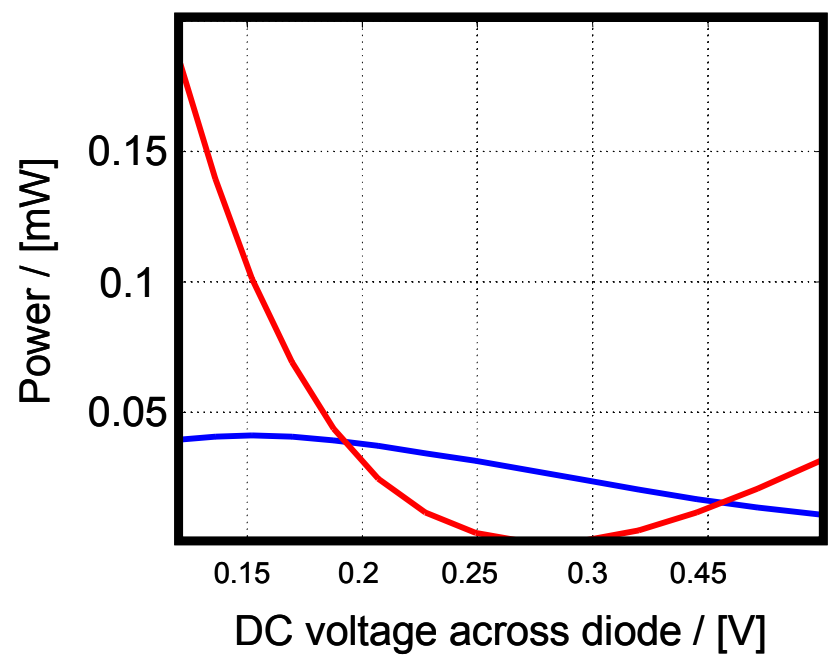

Fig. 7. Model results of the power in the carrier (red) and either of the nearest first side band (blue) of the reflected signal from the RF port of the tunnel diode. For this particular choice of RF input power and modulation amplitude the carrier is completely suppressed with around $0.28 \mathrm{~V}$ DC bias applied.

DC level is adjusted in an iterative procedure to null the diode current. This is done to simulate an open termination of the IF port. As can be seen, there is a reasonable correspondence between numerical and experimental results and both show a sensitivity above $1200 \mathrm{mV} / \mathrm{mW}$ for an input power of $0.1 \mathrm{~mW}$. The experimental results were obtained at $10 \mathrm{GHz}$. The performance of the diode as modulator is illustrated in Fig. 6 where the power in one of the first side bands around the carrier is shown versus input power in the RF carrier and at various peak-to-peak modulation voltages. Highest Pound sensitivity, with a constant input power to the resonator occurs for a PM modulation index of roughly 1 . Here, the power in the carrier is around twice the power in the first side bands so given the results from Fig. 6 it should be possible to send around $-10 \mathrm{dBm}$ to the resonator. With a loaded quality factor of around 70 million and a coupling factor of around 0.2 of the sapphire resonator, and the results on the sensitivity and modulation ability of the tunnel diode, we predict a Pound sensitivity, measured at the tunnel diode output, of $0.1-0.2 \mathrm{mV} / \mathrm{Hz}$. This is very good, as our noise analysis in a later section will show, and thus, further development of a cryogenic Pound circuit based on tunnel diodes is justified.

\section{B. Carrier Suppression}

In the design of the phase modulator a pair of polarity inverted diodes were used initially in a symmetric configuration. The reflected AM modulated signals were combined in a $3 \mathrm{~dB}$ hybrid. Due to the polarity inversion of the diodes the dark port of the hybrid showed a reduced carrier. By adjusting the common DC bias to the diodes applied along with the modulation signal at the IF ports, the

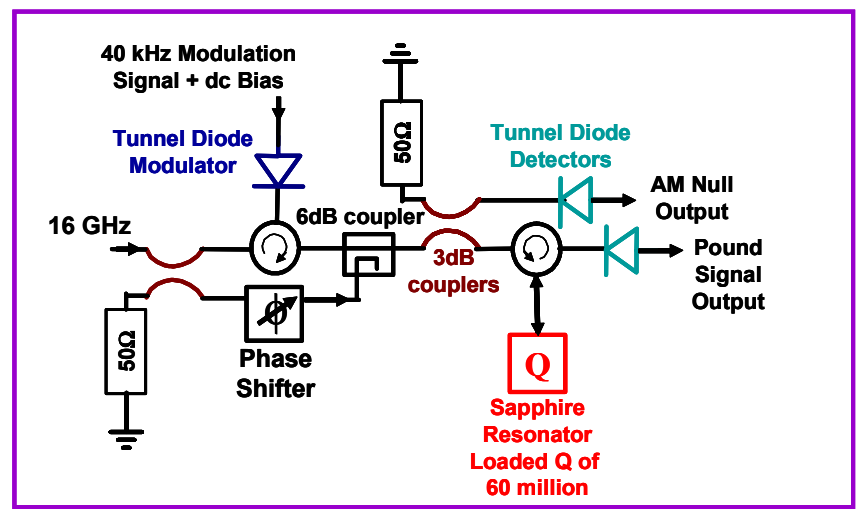

Fig. 8. The new cryogenic Pound circuit. The tunnel diode modulator is DC biased and the phase shifter adjusted so that the upper diode detector gives minimal output at the same time as having high power in the modulation sidebands. The power in the sidebands is monitored through a weakly coupled port not shown in the diagram inserted immediately to the right of the left circulator. The carrier signal (here $16 \mathrm{GHz}$ ) thus enters the cryogenic environment, gets phase modulated and the phase modulated signal is reflected from the resonator. In general this reflection contains an AM component which is detected in a tunnel diode, thus providing the Pound signal.

carrier could be removed entirely. However, the required DC bias to null the carrier had a reducing effect on the side bands.

The second design of the phase modulator using just one diode was created based on the observation that by adjusting the DC bias to the diode, the reflected signal could show almost total carrier suppression regardless of input RF power level, and modulation amplitude. This is also predicted by the model. In Fig. 7, the power in the carrier and the first side band of the reflected signal is shown as a function of the DC bias. At around $280 \mathrm{mV}$ the carrier is removed completely. The physical explanation for this phenomenon is the phase inversion that takes place when the dynamic resistance of the diode crosses the characteristic impedance of the transmission line $Z_{0}(=50 \Omega$ in our case).

\section{Cryogenic Pound Circuit Topology}

The second design of the carrier suppression was chosen for the cryogenic Pound circuit since it only uses one diode and actually gives the best overall performance. The schematic design of the Pound circuit can be seen in Fig. 8. An RF carrier (16 GHz here) corresponding to the resonance frequency of the resonator is transmitted to the cryogenic environment. Here, it gets divided into two branches in a $3 \mathrm{~dB}$ hybrid. The upper branch sends the carrier to the modulator diode through a circulator. The DC level is adjusted to give carrier suppression so the signal that is reflected only contains all the side bands. The carrier in the lower branch is phase shifted mechanically before it is injected into the upper branch containing the reflection from the diode. The sum signal is now the phase modulated carrier. This signal is further divided in two in a $3 \mathrm{~dB}$ hybrid, 


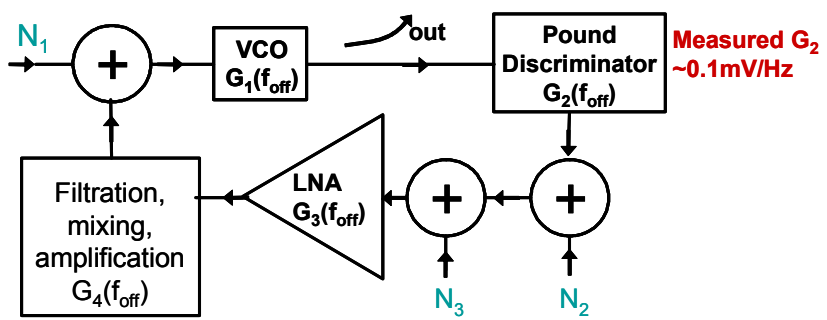

Fig. 9. Diagram of the oscillator for the purpose of doing noise and stability analysis.

where one coupled port is connected directly to a tunnel diode detector and the signal in the second coupled port is sent to the resonator. The directly coupled diode detects any residual AM component that would give rise to a false Pound response, and it is used in a servo circuit to maintain the required DC bias level for the modulator diode. Before this servo loop is closed the mechanical phase shifter is adjusted to give a minimal signal from the directly coupled diode detector. Since the phase shifter is inaccessible in the cryogenic environment, one cool-down where the Pound circuit is split in a cold part and a part at room-temperature that include the phase shifter is required. Thus, the phase can be adjusted before the phase shifter is placed in the cold environment in the next cool down.

The measured sensitivity of the new cryogenic Pound circuit was $0.1 \mathrm{mV} / \mathrm{Hz}$ which is in accordance with the estimated result based on the performance as modulator and detector of the tunnel diode.

\section{Noise Analysis}

In Fig. 9 a diagram of an oscillator using a Pound discriminator is shown. The VCO is the oscillator that we wish to stabilize with the Pound circuit. $N_{1}, N_{2}$ and $N_{3}$ represent the input voltage noise of the $\mathrm{VCO}$, the voltage noise in the discriminator and the input noise of the LNA that amplifies the Pound signal. $N_{2}$ is the composite noise generated by noise in the DC bias of the modulator diode, noise in the passive devices, temperature variations of the resonator, noise in the tunnel diodes and RF power level fluctuations. The DC bias noise is highly suppressed by the servo loop that maintains the appropriate DC level of the modulator. We have chosen this loop to be fast (unity gain at around $1 \mathrm{kHz}$ ) compared with the Pound stabilizer electronics (unity gain at around $30 \mathrm{~Hz}$ ). The noise in tunnel diodes is very small and is assumed zero in the following analysis. A direct measurement of the diode noise at the various different DC bias levels applied is, however, planned in the investigation of why the stability predicted in this section is not seen experimentally. With very careful design of the electronics we believe that the overall noise performance of the Pound discriminator will be better than the input stage of the LNA. We are therefore left with $N_{l}$

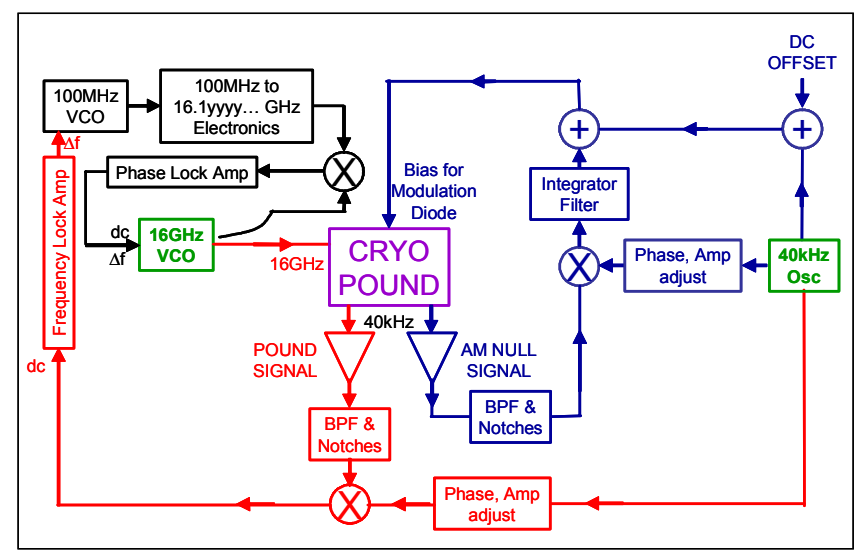

Fig. 10. Diagram of the oscillator using the cryogenic Pound circuit to stabilize a $100 \mathrm{MHz}$ VCO (VCXO). The black loop phase locks the $16 \mathrm{GHz}$ DRO to the $100 \mathrm{MHz}$ VCO. The blue loop is the servo circuit that maintains the DC bias across the modulator diode in order to null the AM component in the modulated $16 \mathrm{GHz}$ signal that is sent to the resonator. The Pound loop is colored red.

and $N_{3}$. These noise sources give rise to frequency variations $\Delta f_{l}$ and $\Delta f_{3}$, respectively:

$$
\Delta f_{1} \approx \frac{N_{1}}{G_{2} G_{3} G_{4}},
$$

and

$$
\Delta f_{3} \approx \frac{N_{3}}{G_{2}} .
$$

With the high gain in the LNA of 20-30 dB the realizable frequency stability of the VCO will be limited by $N_{3}$, which is therefore assumed to be the only noise source in the following, i.e. the total frequency variation $\Delta f \approx \Delta f_{3}$.

The normalized frequency departure $y(t)$ from the VCO nominal frequency $f_{0}$ is

$$
y(t)=\frac{\Delta f}{f_{0}} \approx \frac{N_{3}}{f_{0} G_{2}},
$$

and thus, the one-sided spectral densities $S_{y}$ of $y$ and $S_{N 3}$ of $N_{3}$ are related through:

$$
S_{y}(f) \approx\left[\frac{1}{f_{0} G_{2}}\right]^{2} S_{N_{3}}(f) .
$$

The frequency of the relevant part of the Pound signal is the modulation frequency. If the LNA is not run into compression, there will be minimal up-conversion of the 
flicker noise in the LNA, so it is sufficient to insert the white noise of the LNA in the above expression. We use the LT1028 from Linear Technology in the input stage which has the input voltage noise corresponding to a $50 \Omega$ resistor:

$$
S_{y}(f) \approx\left[\frac{1}{16 G H z \cdot 0.1 \frac{\mathrm{mV}}{\mathrm{Hz}}}\right]^{2} 4 k T 50 \Omega=3 \cdot 10^{-31}
$$

From [8], we estimate the Allan deviation from $S_{y}$

$$
\sigma_{y}(\tau) \approx \sqrt{\frac{S_{y}}{2 \tau}}=4 \cdot 10^{-16} / \sqrt{\tau}
$$

This is really good and more than an order of magnitude more stable than what has previously been achieved with the resonator used here [9].

\section{E. Experimental}

We use the same sapphire resonator as in [9]. The turnover temperature is around $37 \mathrm{~K}$. The sapphire parts are maintained at the turnover temperature and the cavity is controlled at a temperature approximately $1 \mathrm{~K}$ higher. The sapphire parts are isolated from the Copper cavity with a thermal time constant of $2000 \mathrm{~s}$, while the cavity is in good thermal contact with the rest of the Pound circuitry. The temperature of the Pound is therefore very stable i.e. the variation is within a couple of $\mathrm{mKs}$.

The resonance is at around $16.1 \mathrm{GHz}$ and the quality factor approximately 70 million. For these tests, the resonator is coupled through one port with a relatively poor coupling factor of around 0.2 .

$40 \mathrm{kHz}$ was chosen as the modulation frequency which is sufficient for the low-noise $100 \mathrm{MHz}$ VCXO that we used, and the modulator diode was biased at roughly $-1.2 \mathrm{~V}$ where the ability to generate power in the sidebands with good carrier suppression was better than at the positive bias of around $0.3 \mathrm{~V}$. It was found that the diode detector for the residual AM in the signal from the modulator was unstable without a fixed DC bias. This bias was chosen to be $20 \mathrm{mV}$ where an optimum compromise between high RF sensitivity and low DC bias sensitivity was found.

\section{F. Oscillator Performance}

In Fig. 10 a complete diagram of the oscillator is shown. The cryogenic Pound topology introduces the additional loop (AM null loop) to maintain a bias, where the upper detector diode in Fig. 8 gives a minimal signal at the modulation frequency.

The $16 \mathrm{GHz}$ DRO is phase locked to the $100 \mathrm{MHz}$ VCXO and the Pound loop stabilizes the VCXO. The amplifiers of the signals from both detectors in the Pound circuit are built qualitatively in the same manner with a low

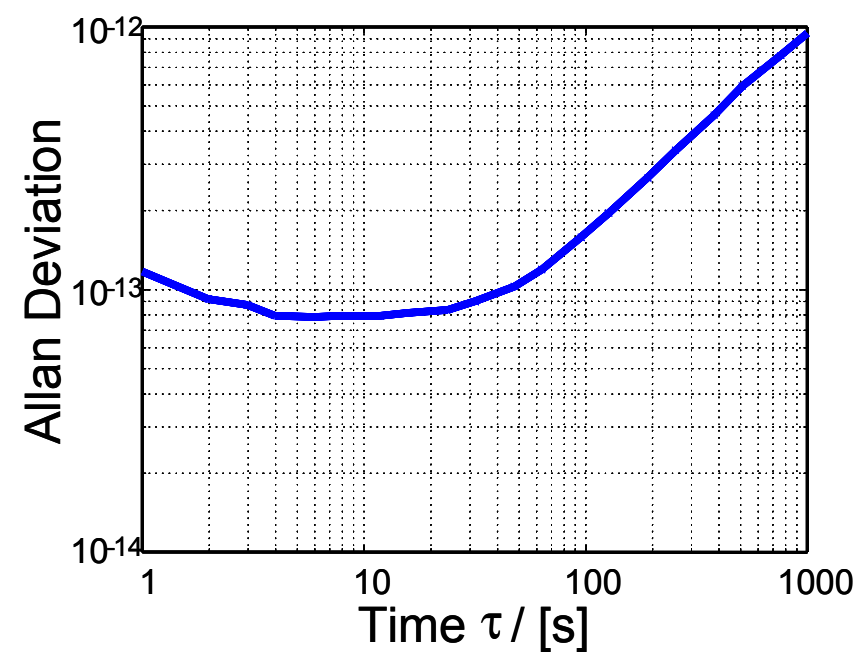

Fig. 11. Initial stability measurement of the oscillator. Measured by beating the $100 \mathrm{MHz}$ stabilized VCO with the best $100 \mathrm{MHz}$ reference at JPL which has a stability performance at least an order of magnitude better than measured here.

noise broad band amplification stage followed by a band pass filter around $40 \mathrm{kHz}$ and notch filters at the three harmonics $(80,120$ and $160 \mathrm{kHz})$. The gains in the two loops are adjusted to give a unity gain point at $37 \mathrm{~Hz}$ of the Pound loop and at $1.5 \mathrm{kHz}$ of the AM null loop.

In Fig. 11 the stability of the oscillator is shown. A reasonable result of $1 \cdot 10^{-13}$ is obtained at 1 second, but this is of course far from what the previous noise analysis predicted. However, we have proved that the cryogenic Pound indeed works. In this first implementation many new components have been introduced and not thoroughly tested, so we are confident that with more experimental investigation a better performance can be achieved.

\section{CONCLUSION}

We have developed a new all-cryogenic Pound circuit using tunnel diodes to phase modulate an RF carrier and as AM detectors. The performance of the Pound discriminator $(0.1 \mathrm{mV} / \mathrm{Hz})$ is in good accordance with model predictions. We have further demonstrated the ability of the circuit to stabilize a $100 \mathrm{MHz}$ VCXO.

The noise analysis of the circuit based on the measured discriminator performance shows that this new loop has the potential of becoming much more stable than the usual Pound implementation since sensitivity to temperature variations in RF transmission lines has been effectively removed. As such, we estimate a possible $10^{-15} / \sqrt{ } \tau$ or better performance of the new Pound circuit. The circuit uses a resonator that has been used in traditional Pound circuits where the stability was an order of magnitude worse than this projection.

The new Pound circuit introduces an extra loop to maintain an appropriate DC bias across the tunnel diode 
used to modulate the carrier. We have designed this loop to be fast compared to the Pound loop to minimize interactions between the two feedback loops. In our first experimental investigations of the all-cryogenic Pound circuit we achieved a stability of $1 \cdot 10^{-13}$ at 1 second, so we are still some way from the predicted possible performance. However, the new topology has introduced many new components in the oscillator each of which if not carefully designed and built can ruin the overall performance, so with more investigation and individual component optimization, we are confident we can improve the stability.

\section{ACKNOWLEDGMENTS}

Lute Maleki is acknowledged for his invitation of R. Basu to work in the Quantum Sciences \& Technology group at the Jet Propulsion Laboratory from August 2004 to September 2005.

This work was carried out at the Jet Propulsion Laboratory, California Institute of Technology, under a contract with the National Aeronautics and Space Administration.

\section{REFERENCES}

[1] R. V. Pound, "Electronic frequency stabilization of microwave oscillators," Rev. Sci. Instrum. 17, 490-505, 1946.

[2] G. J. Dick and R. T. Wang, "Stability and phase noise tests of two cryo-cooled sapphire oscillators," IEEE Trans. UFFC 47 \#5, 1098$1101,2000$.

[3] A. G. Mann, C. Sheng, and A. N. Luiten, "Cryogenic sapphire oscillator with exceptionally high frequency stability," Conference on Precision Electromagnetic Measurements, Sydney, Australia May 2000, 188-189, 2000.

[4] G. J. Dick, "Pound circuit-induced frequency sensitivities in ultrastable cryogenic oscillators," Proceedings of the 2004 IEEE International Frequency Control Symposium, 224-228, 2004.

[5] G. J. Dick, D. G. Santiago, and R. T. Wang, "Frequency stability of $1 \times 10^{-13}$ in a compensated sapphiore oscillator operating above $77 \mathrm{~K}$," Proceedings of the Tenth European Frequency and Time Forum, 262265, 1996.

[6] Tunnel diode DT1218A1 from Eclipse Microwave Inc.

[7] Donald A Neamen, Semiconductor Physics and Devices: Basic Principles, 3rd ed. McGraw-Hill, 2003.

[8] David Allan et al, "Standard terminology for fundamental frequency and time metrology," Proceedings of the $42^{\text {nd }}$ Annual Frequency Control Symposium, 419-425, 1988.

[9] R. T. Wang and G. J. Dick, "High stability 40 Kelvin cryo-cooled sapphire oscillator," Proceedings of the 2003 IEEE International Frequency Control Symposium, 371-375, 2003. 